\title{
Assessing Heterogeneity in the Relationship Between Wheat Yield and Fusarium Head Blight Intensity Using Random-Coefficient Mixed Models
}

\author{
L. V. Madden and P. A. Paul
}

Department of Plant Pathology, The Ohio State University, Wooster 44691.

Accepted for publication 23 February 2009.

\begin{abstract}
Madden, L. V., and Paul, P. A. 2009. Assessing heterogeneity in the relationship between wheat yield and Fusarium head blight intensity using random-coefficient mixed models. Phytopathology 99:850-860.

Seventy-seven studies reporting Fusarium head blight disease index ( $Y$; mean percentage of diseased spikelets per spike) and wheat yield ( $W$; $\mathrm{MT} / \mathrm{ha}$ ) were analyzed to determine the relationship between $W$ and $Y$, and to assess the degree of variation for the relationship among studies. A linear random-coefficient model-comprising a population-average intercept and slope, a random residual term, and random effects of study on the intercept and slope (best linear unbiased predictors; BLUPs) - was successfully fitted to the data using maximum likelihood. From the predicted random effects, study-specific intercepts and slopes were obtained, and both population-average and subject-specific predictions of yield

slope, with soft-red winter wheat having, on average, $0.85 \mathrm{MT} / \mathrm{ha}$ higher yield than spring wheat. Based on the estimates of the among-study variances, there was high variation in the effects of study on the intercept, but substantially lower variation in the effects of study on the slope. Thus, although one cannot predict with accuracy the actual wheat yield in a field or plot based on disease index using population-average results, one can predict with accuracy the decline in yield at a given level of disease index using the population-average slope. Through the modeling results, predicted relative yield (as a percentage of yield when disease is not present) can be determined, as well as predicted disease index at which a prespecified level of yield (or yield loss) is expected to occur. The predicted reduction in yield on a percentage scale was greater for spring than for soft-red winter wheat, on average, because of the lower estimated intercept in absolute units for spring wheat.
\end{abstract} were determined. The estimated population-average intercept (expected yield when disease symptoms were not present) was $4.10 \mathrm{MT} / \mathrm{ha}$, and the population-average slope was $0.038 \mathrm{MT} / \mathrm{ha}$ per unit increase of disease index. Wheat class had a significant effect on the intercept but not on the
Additional keywords: empirical Bayes model, hierarchical linear model, linear mixed model, studentized residual plot.
Plant disease epidemics continue to result in reductions in quantity and quality of crop yield throughout the world $(7,16,30$, 32). Moreover, the short- and long-term costs of managing diseases reduce profits for growers and place an economic burden on society as a whole $(7,43)$. Many studies have characterized the relationship between crop yield ( $W$; weight, biomass, or volume per unit area) and attributes of epidemics (Chapter 12 in citation 16). These attributes include disease intensity at one or more particular times; "virtual lesion area" at a particular time (1); time of infection and corresponding disease progress rates (15); area under the disease progress curve; healthy area (green leaf) duration and integration of radiation intercepted (or absorbed) by healthy plant tissue and radiation use efficiency (38). Various models, both empirical and mechanistic, have been developed to relate $W$ to epidemic attributes $(8,32,36)$. By far, the most common epidemic attribute is disease intensity at a single time $(13,16)$.

Few investigations have been conducted to quantify formally the degree of variability or heterogeneity in the relationship between crop yield and epidemic attributes. This is partly because few researchers can afford to conduct a sufficient number of studies to obtain precise estimates of the variability in the relationship or precise estimates of the parameters of models for the yield-disease relationship. As a consequence, it is often difficult

Corresponding author: L. V. Madden; E-mail address: MADDEN.1@ osu.edu

doi:10.1094/PHYTO-99-7-0850

This article is in the public domain and not copyrightable. It may be freely reprinted with customary crediting of the source. The American Phytopathological Society, 2009. to know if an observed relationship is 'typical', or if there is high or low certainty in the parameter estimates obtained in a given study or in a small number of studies.

The goal of our current work is to characterize explicitly the expected relationship between yield and disease intensity, as well as the heterogeneity in the relationship, for Fusarium head blight (FHB) of wheat $(17,34,35)$. To meet this goal, we utilized random-coefficient mixed models because these models are well suited for estimating parameters for expected relationships between variables while simultaneously estimating variance and covariance terms for the heterogeneity of results at multiple scales in a hierarchy $(10,12)$. Because the selected statistical methodology is uncommon in plant pathology and crop loss assessment, we describe random-coefficient modeling in detail in the Materials and Methods.

FHB, also known as wheat scab or ear blight, may be the most economically important crop disease in the last 50 years in the United States (40). The disease is caused by Gibberella zeae (anamorph: Fusarium graminearum), as well as other fungi (17, 23,35). In the United States, however, G. zeae is the dominant pathogen species for this monocyclic disease. Epidemics are driven by the magnitude of inoculum density and regulated by environmental conditions (34). Infection occurs during a narrow time window, starting around anthesis (Feekes growth stage 10.5.1 [9] or Zadoks scale 60 [42]). Yield loss occurs due to direct death of flowers (resulting in fewer grains being produced per spike), as well as to smaller size and weight of the grains produced (17). In addition to reductions in yield, epidemics are often associated with the accumulation of the toxin deoxynivalenol (DON) in harvested grain. The price obtained per unit volume 
or weight of harvested grain depends on the concentration of DON, test weight, and percentage of Fusarium-damaged kernels (17).

Although there are numerous reports on the economic impact of FHB on wheat and other grains $(17,23,39)$, the functional relationship between yield and symptoms of the disease is not well understood (23). As part of the U.S. Wheat and Barley Scab Initiative, Uniform Fungicide Trials (UFTs) have been conducted using standardized methods for over a decade specifically to determine the influence of different fungicide treatments on disease intensity in the field and DON concentration in harvested grain $(26,27)$. Grain yield was recorded in several of these studies, however, and these data can provide information on direct crop losses due to FHB in wheat. Therefore, we utilized data obtained from the UFTs to characterize the relationship between yield and disease intensity as well as the heterogeneity in the relationship.

\section{MATERIALS AND METHODS}

Data collection. Fungicide studies were conducted in fields across the U.S. soft winter wheat-producing region (Mid-Atlantic, southern, and Midwestern regions not including Iowa, Minnesota and the Dakotas) and spring wheat-producing region (Iowa, Minnesota, and the Dakotas) between 1998 and 2007. These studies comprise the UFTs, which were described in detail previously $(26,27)$. Results from these trials and an additional nine trials conducted with the same protocols between 1995 and 1997 (prior to the establishment of the national UFTs, but which served as the basis for the national UFTs) were considered in this investigation. The studies conducted before 2006 previously were utilized in several meta-analyses to determine the effects of fungicides on control of FHB and DON, and for characterizing the relationship between DON and FHB intensity (26-29).

Studies generally were conducted according to a standard protocol, using a susceptible and/or moderately susceptible cultivar and agronomic practices appropriate for each location. Plots were either planted into corn or wheat residue, or artificially infested with $F$. graminearum-infested kernels and mist-irrigated as a means of enhancing inoculum production, infection, and disease development. There were typically from four to seven treatments in each study, including the untreated control, although some studies had as many as 11 treatments. Fungicides (typically of the triazole class) were applied at Feekes growth stage 10.5.1 (Zadoks scale 60). The specific fungicide treatments, and their application rates, varied from year to year (27).

Disease intensity was determined as the mean percentage of diseased spikelets per spike, known in the FHB literature as disease index, or field or plot severity $(24,26)$. Disease index was assessed at Feekes growth stage 11.2 (Zadoks scale 85-soft dough), usually by observing 20 to 50 spikes per plot. Plots were harvested using a research-plot combine and average yield was determined for each treatment. Yield data were available for at least some treatments in 136 of the 201 studies conducted between 1995 and 2007.

To determine the relationship between yield and index, a range of index values must be obtained in a study. Thus, a study was only included in the current analysis if the range in mean disease index from the largest to the smallest value was greater than $2 \%$. Furthermore, both index and yield had to be determined in the (untreated) control treatment for a study to be included. These criteria eliminated studies where no or little evidence of disease symptoms was recorded. Using this criterion, 77 studies were utilized in the described analyses. Although yield was originally recorded as bushels per acre (a volumetric measurement per unit area), it was converted to metric tons per hectare (MT/ha). The conversion was done using a test weight of $25 \mathrm{~kg} / \mathrm{bushel}$. This weight per volume was determined by calculating the mean from test-weight data obtained from 121 studies.
Data analysis-simple linear models. We fitted a linear statistical model $(16,33)$ to the yield-disease data for each study. For a single study, the model is of the form

$$
W_{j}=\beta_{0}-\beta_{1} Y_{j}+e_{j}
$$

in which the $j$ subscript refers to the $j$ th observation $(j=1, \ldots, n)$ in a study, $W_{j}$ is yield (MT/ha) for observation $j, Y_{j}$ is disease index $(\%)$ at Feekes growth stage 11.2 (Zadoks scale 85) for observation $j$, and $\beta_{0}$ and $\beta_{1}$ are parameters. $\beta_{0}$ represents the expected yield when disease index is 0 (units of metric tons per hectare; MT/ha), and $\beta_{1}$ is the decline in expected yield with unit increase in $Y$ (units of metric tons per hectare per unit increase in disease index; MT $\mathrm{ha}^{-1} \%^{-1}$ ). Note that in this formulation, a positive $\beta_{1}$ indicates that yield declines with increasing disease. $e_{j}$ is the residual for observation $j$, the difference between observed yield and that expected for given values of the parameters $\left(e_{j}=W_{j}-\right.$ $\left.\left[\beta_{0}-\beta_{1} Y_{j}\right]\right)$; it is assumed that the residual is normally distributed with mean 0 and variance $\sigma_{e}^{2}$.

For multiple studies, equation 1a can be expanded to

$$
W_{i j}=\beta_{0 i}-\beta_{1 i} Y_{i j}+e_{i j}
$$

in which the $i$ subscript represents the individual study, and $j$ subscript represents the individual observation within study $i, W_{i j}$, $Y_{i j}$, and $e_{i j}$ represent yield, disease index, and the residual, respectively, for the $j$ th observation of the $i$ th study, and $\beta_{0 i}$ and $\beta_{1 i}$ are parameters. In equation $1 \mathrm{~b}$, there is a separate intercept and slope for each study (as indicated by the $i$ subscript). The expected yield for study $i$ is given by $\beta_{0 i}-\beta_{1 i} Y_{i j}$. A special case of equation $1 \mathrm{~b}$ is when the intercept and slope do not vary across studies (so that $\beta_{0}$ and $\beta_{1}$ are substituted for $\beta_{0 i}$ and $\beta_{1 i}$, respectively).

The MIXED procedure of SAS (SAS Institute Inc., Cary, NC) was used to fit equation $1 \mathrm{~b}$ to the data using maximum likelihood. Designating the estimated parameters for the different studies as $\hat{\beta}_{0 i}$ and $\hat{\beta}_{1 i}$, the predicted yield at a given level of disease index in a study, which is an estimate of the expected yield for that study (33), is written as

$$
\hat{W}_{i j}=\hat{\beta}_{0 i}-\hat{\beta}_{1 i} Y_{i j}
$$

The estimated residual is given by $\hat{e}_{i j}=W_{i j}-\hat{W}_{i j}$.

To summarize the distribution of estimated intercepts and slopes, percentiles of the $77 \hat{\beta}_{0 i}$ and $\hat{\beta}_{1 i}$ values were determined, such as the $10^{\text {th }}\left(p_{10}\right), 50^{\text {th }}$ (median), and $90^{\text {th }}\left(p_{90}\right)$ percentiles. The difference of $p_{90}$ and $p_{10}$, labeled the interdecile range (ID $=p_{90}-p_{10}$ ), was calculated as a robust measure of the spread (variability) of the parameter estimates. ID encompasses $80 \%$ of the estimated intercept or slope values.

Data analysis-random coefficients models. In a randomcoefficient model, equations $1 \mathrm{a}$ and $1 \mathrm{~b}$ are expanded to account for the random effect of each study on the intercept and slope $(10,12,41)$. The model can be written as

$$
W_{i j}=\left(\beta_{0}+u_{0 i}\right)-\left(\beta_{1}+u_{1 i}\right) Y_{i j}+e_{i j}
$$

in which $u_{0 i}$ is the effect of the $i$ th study on the intercept and $u_{1 i}$ is the effect of the $i$ th study on the slope. Both $u_{0 i}$ and $u_{1 i}$ are random variables, with mean 0 and variances $\sigma_{u_{0}}^{2}$ and $\sigma_{u_{1}}^{2}$, respectively. The covariance between $u_{0 i}$ and $u_{1 i}$ is represented by $\sigma_{u_{0}, u_{1}}$. It is assumed that the within-study, or residual, variance of the $e_{i j}\left(\sigma_{e}^{2}\right)$ is independent of $u_{0 i}$ and $u_{1 i}$; this is the standard assumption for random-coefficient models (10). The distributional assumptions of equation 2 can be written succinctly as

$$
\begin{aligned}
\left(\begin{array}{l}
u_{0 i} \\
u_{1 i}
\end{array}\right) & \sim N\left(\left(\begin{array}{l}
0 \\
0
\end{array}\right),\left(\begin{array}{cc}
\sigma_{u_{0}}^{2} & \sigma_{u_{0}, u_{1}} \\
\sigma_{u_{0}, u_{1}} & \sigma_{u_{1}}^{2}
\end{array}\right)\right) \\
e_{i j} & \sim N\left(0, \sigma_{e}^{2}\right)
\end{aligned}
$$


A random-coefficient model is one specific form of a mixed model (5), and is also known as an empirical Bayes model, a hierarchical linear model, and a multilevel model $(4,10)$. As stated by de Leeeuw and Kreft (4), although random-coefficient models are of value under many circumstances, they are especially useful when there is a relatively large number of 'subjects' (studies in our case) and a relatively small number of observations for each 'subject'. This is the situation with our investigation. In equation $2, \beta_{0}$ and $\beta_{1}$ are known as the population-average intercept (MT/ha) and slope (MT ha ${ }^{-1} \%^{-1}$ ), respectively, reflecting the overall relationship between yield and disease index. The $u_{0 i}$ and $u_{1 i}$ variables are known as the study-specific random effects of the $i$ th study on the intercept and slope, respectively. In a statistically technical sense, each $u$ term is a best linear unbiased prediction (BLUP) of the study effect (10).

Equation 2 can be rewritten as

$$
W_{i j}=b_{0 i}-b_{1 i} Y_{i j}+e_{i j}
$$

with $b_{0 i}=\beta_{0}+u_{0 i}$ and $b_{1 i}=\beta_{1}+u_{1 i}$. Here, $b_{0 i}$ and $b_{1 i}$ are the studyspecific intercepts and slopes, respectively, where each $b$ term is comprised of a population-average coefficient $\left(\beta_{0}\right.$ or $\left.\beta_{1}\right)$ and a random effect of the individual study $\left(u_{0 i}\right.$ or $\left.u_{1 i}\right)$ on the populationaverage coefficient. The $b_{0 i}$ and $b_{1 i}$ values can be considered as random samples from a population of intercepts and slopes, respectively; the $b_{0 i}$ and $b_{1 i}$ terms are also BLUPs. Although equations $1 \mathrm{~b}$ and 4 both specify a separate intercept and slope for each study, only equation 4 treats these as random variables.

The expected (mean) yield as a function of disease index can be obtained from equation 2. The unconditional or marginal expected yield is given by

$$
E\left(W_{i j}\right)=\beta_{0}-\beta_{1} Y_{i j}
$$

which is a population-average expectation (across all studies). The $u_{0 i}$ and $u_{1 i}$ variables disappear in the unconditional-expectation operation because these variables both have means of 0 . The conditional expected yield is given by

$$
E\left(W_{i j} \mid u_{0 i}, u_{1 i}\right)=\left(\beta_{0}+u_{0 i}\right)-\left(\beta_{1}+u_{1 i}\right) Y_{i j}=b_{0 i}-b_{1 i} Y_{i j}
$$

Equation $5 \mathrm{~b}$ is the study-specific expectation of yield, indicating the mean yield for a given disease index for each individual study.

Equation 2 was fitted to the data using maximum likelihood (ML). The model fitting procedure results in estimates of $\beta_{0}\left(\hat{\beta}_{0}\right)$ and $\beta_{1}\left(\hat{\beta}_{1}\right)$, as well as the estimates of the variance components and covariance in equation 3 . Type $3 t$ tests were performed to determine if the intercept or slope were different from 0 . Moreover, predictions of $u_{0 i}\left(\hat{u}_{0 i}\right)$ and $u_{1 i}\left(\hat{u}_{1 i}\right)$ were obtained from the mixed-model solution equations. (Because the effects of study on the intercept and slope are random variables, in a technical sense they are predicted and not estimated). Model fitting was done using the MIXED procedure of SAS. In parallel to the unconditional and conditional expectations of yield (equations $5 \mathrm{a}$ and $5 \mathrm{~b}$ ), the unconditional (marginal) and conditional predictions of yield can be written, respectively, as

$$
\hat{W}_{i j}=\hat{\beta}_{0}-\hat{\beta}_{1} Y_{i j}
$$

and

$$
\hat{W}_{i j} \mid u_{0 i}, u_{1 i}=\left(\hat{\beta}_{0}+\hat{u}_{0 i}\right)-\left(\hat{\beta}_{1}+\hat{u}_{1 i}\right) Y_{i j}
$$

Equation 6a gives the population-average prediction, and equation $6 \mathrm{~b}$ gives the study-specific prediction. Equation $6 \mathrm{~b}$ can be written equivalently as

$$
\hat{W}_{i j} \mid u_{0 i}, u_{1 i}=\hat{b}_{0 i}-\hat{b}_{1 i} Y_{i j}
$$

where $\hat{b}_{0 i}=\hat{\beta}_{0}+\hat{u}_{0 i}$ and $\hat{b}_{1 i}=\hat{\beta}_{1}+\hat{u}_{1 i}$.

Unconditional or marginal residuals $\left(\hat{e}_{i j}=W_{i j}-\hat{W}_{i j}\right)$ and conditional residuals $\left(\hat{e}_{i j}\left|u_{0 i}, u_{1 i}=W_{i j}-\hat{W}_{i j}\right| u_{0 i}, u_{1 i}\right)$, as well as the unconditional and conditional studentized residuals, were estimated. The studentized residual were evaluated graphically to determine the appropriateness of the random-coefficient model fit (10,33). Both the unconditional and conditional studentized residuals are relevant, depending on whether interest is on inference for the population-average or individual studies. The ID was calculated as a measure of spread or variability of the subject-specific predicted intercepts $\left(\hat{b}_{0 i}\right)$ and slopes $\left(\hat{b}_{1 i}\right)$.

For mixed models in general, there is no natural and unambiguous statistic - such as the usual coefficient of determination $\left(R^{2}\right)$ — to measure the goodness of fit on a unitless scale (e.g., 0 to 1 or 0 to $100 \%)(11,37)$. Rather, for mixed models, statistics such as the log-likelihood are used to compare the goodness of fit of one model relative to another. However, several $R^{2}$-type statistics have been developed that have some properties similar to the $R^{2}$. We assessed the goodness of fit of the population-average predictions (equation 6a) to the data using the $R_{F}{ }^{2}$ statistic of Liu et al. (11), and the fit of the study-specific predictions (equation 6b) to the data using the $R_{T}^{2}$ statistic of the same authors. Furthermore, we use the concordance correlation coefficient $(16,37)$ as a further measure of fit. The interpretation of this latter statistic for the fit of mixed models is given in Paul et al. (25).

Data analysis - model expansions. Equation 4 was expanded to account for the effect of wheat type on the relationship between yield and disease index. We considered wheat type to be a categorical variable (factor), with two levels indexed by the $k$ subscript. If we let $\xi_{k}$ represent the effect of wheat type on the intercept and $\zeta_{k}$ represent the effect of wheat type on the slope, the random-coefficient model is written as

$$
W_{i j k}=\left(\beta_{0}+\xi_{k}+u_{0 i}\right)-\left(\beta_{1}+\varsigma_{k}+u_{1 i}\right) Y_{i j k}+e_{i j k}
$$

There are three subscripts now on $W$ (or $Y$ ), to represent yield (or disease) for the $j$ th observation of the $i$ th study of the $k$ th wheat type ( $k=$ 'Spring', 'Winter'). Equation 7 was fitted to the data using ML. Type $3 t$ tests were performed to determine if wheat type significantly affected the intercept or slope (33).

Model expansion also provided an additional mechanism to assess the fit of the equation 2 (or 7) to the data. Disease index was considered both a continuous variable $(Y)$ and a categorical (factor) variable. We define four levels to the disease factor: $d=1$ if $Y$ was between 0 and $7.5 \%$; $d=2$ if $Y$ was between 7.5 and $15 \% ; d=3$ if $Y$ was between 15 and $22.5 \%$; and $d=4$ if $Y$ was greater than $22.5 \%$. Starting with equation 2 , as an example, the generalized model equation can be written as

$$
W_{i j d}=\left(\beta_{0}+u_{0 i}\right)-\left(\beta_{1}+u_{1 i}\right) Y_{i j d}+\Delta_{d}+e_{i j d}
$$

in which $\Delta_{d}$ is the effect of the $d$ th level of disease index on yield. After fitting equation 8 to the data (using ML), a Type $1 F$ test was used to determine if $\Delta$ was significant after the other terms were already in the model (section 8.4.3 in citation 10 for a similar analysis). A significant test result would be evidence that there was some nonlinear, but systematic, variation in yield with increasing index.

The goodness of fit of the population-average predictions and study-specific predictions to the observed yield data were assessed with the $R_{F}{ }^{2}$ and $R_{T}^{2}$ statistics of Liu et al. (11), as well as with the concordance correlation coefficient (37).

Appropriateness of random effects. The Akaike Information Criterion (AIC), which is a function of the log-likelihood, was used to determine whether the random effect portion of the 
selected model could be simplified (10). That is, the AIC was used to see if the data supported a nonzero covariance $\left(\sigma_{u_{0}, u_{1}}\right)$ or nonzero among-study variances $\left(\sigma_{u_{0}}^{2}\right.$ or $\sigma_{u_{1}}^{2}$ ) for the random effects of study on the intercept and slope $(2,10,41)$. If both $\sigma_{u_{0}}^{2}$ and $\sigma_{u_{1}}^{2}$ are 0 , for instance, then random-coefficient equation 2 reduces to the simple linear regression model of equation 1a (a single intercept and slope for all studies). If $\sigma_{u_{1}}^{2}$ is zero, then there is no $u_{1 i}$ term, and equation 2 reduces to a model with a single slope for all studies (but different study-specific intercepts). Likewise, if $\sigma_{u_{0}}^{2}$ is zero, then there is no $u_{0 i}$ term, and equation 2 reduces to a model with a single intercept for all studies (but different study-specific slopes). Comparisons were done by fitting the model with different constraints on the random-effect terms (e.g., 0 covariance, or 0 for one of the variances), and calculating the AIC statistics. The model with the smallest AIC is considered the most appropriate one (of the models being considered) (10).

\section{RESULTS}

Simple linear model. FHB disease index in the (untreated) control plots ranged from 2.1 to $54.7 \%$ across the 77 studies (Fig. 1A). Eighty percent of the values of index were between 5.0 and $35.9 \%$, giving an ID $=30.9 \%$. The maximum yield per study ranged from 1.6 to $6.8 \mathrm{MT} / \mathrm{ha}$ (Fig. 1B), with an ID of 3.8 MT/ha.

The fit of the simple linear equation $1 \mathrm{~b}$ to the data resulted in a wide range of estimated intercepts and slopes (Fig. 2A). The esti-
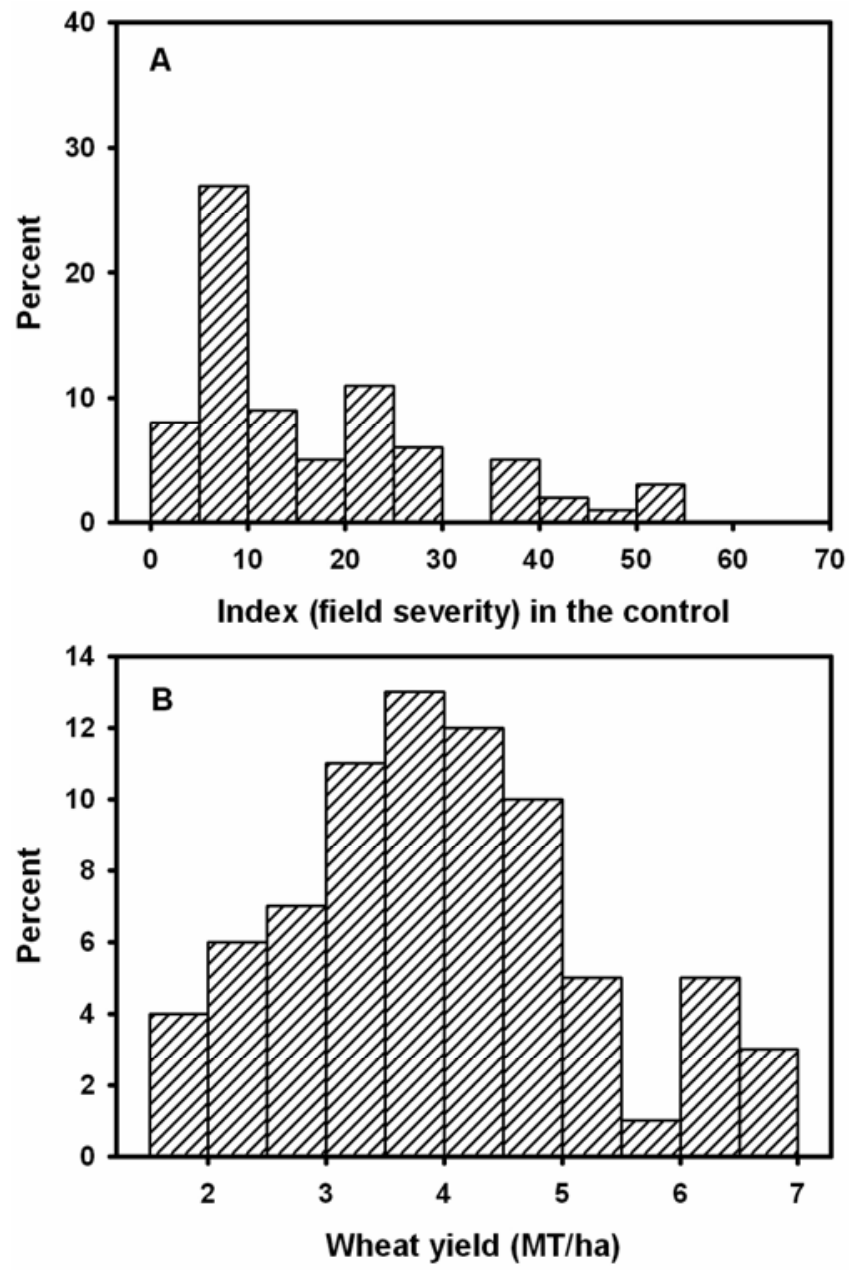

Fig. 1. Histograms of A, Fusarium head blight field severity (disease index; $\%$ ) in the (untreated) control treatment, and B, yield (MT/ha) in the treatment with the highest yield, for 77 studies of the Uniform Fungicide Trials for Fusarium head blight control in wheat. mated intercepts $\left(\hat{\beta}_{0 i}\right)$, corresponding to the predicted yield when disease index was 0 , ranged from 1.8 to $7.2 \mathrm{MT} /$ ha (Fig. $2 \mathrm{~B}$ ), with an overall mean of $4.1 \mathrm{MT} / \mathrm{ha}$ and an ID of $3.3 \mathrm{MT} / \mathrm{ha}$. The estimated slopes $\left(\hat{\beta}_{1 i}\right)$ ranged from -0.13 to $0.29 \mathrm{MT} \mathrm{ha}^{-1} \%{ }^{-1}$ (Fig. 2C), with a mean of 0.053 and an ID of 0.17 . The estimated slopes were negative in $10 \%$ of the studies, suggesting (with little reliability) that yield increased with increasing disease in those studies, the opposite of what would be expected. However, the use of simple equation $1 \mathrm{~b}$ considers each study in isolation and ignores the random effect of study on the parameters; that is, equation $1 \mathrm{~b}$ ignores the fact that each relationship (intercept and slope) is a sample from a population of relationships.
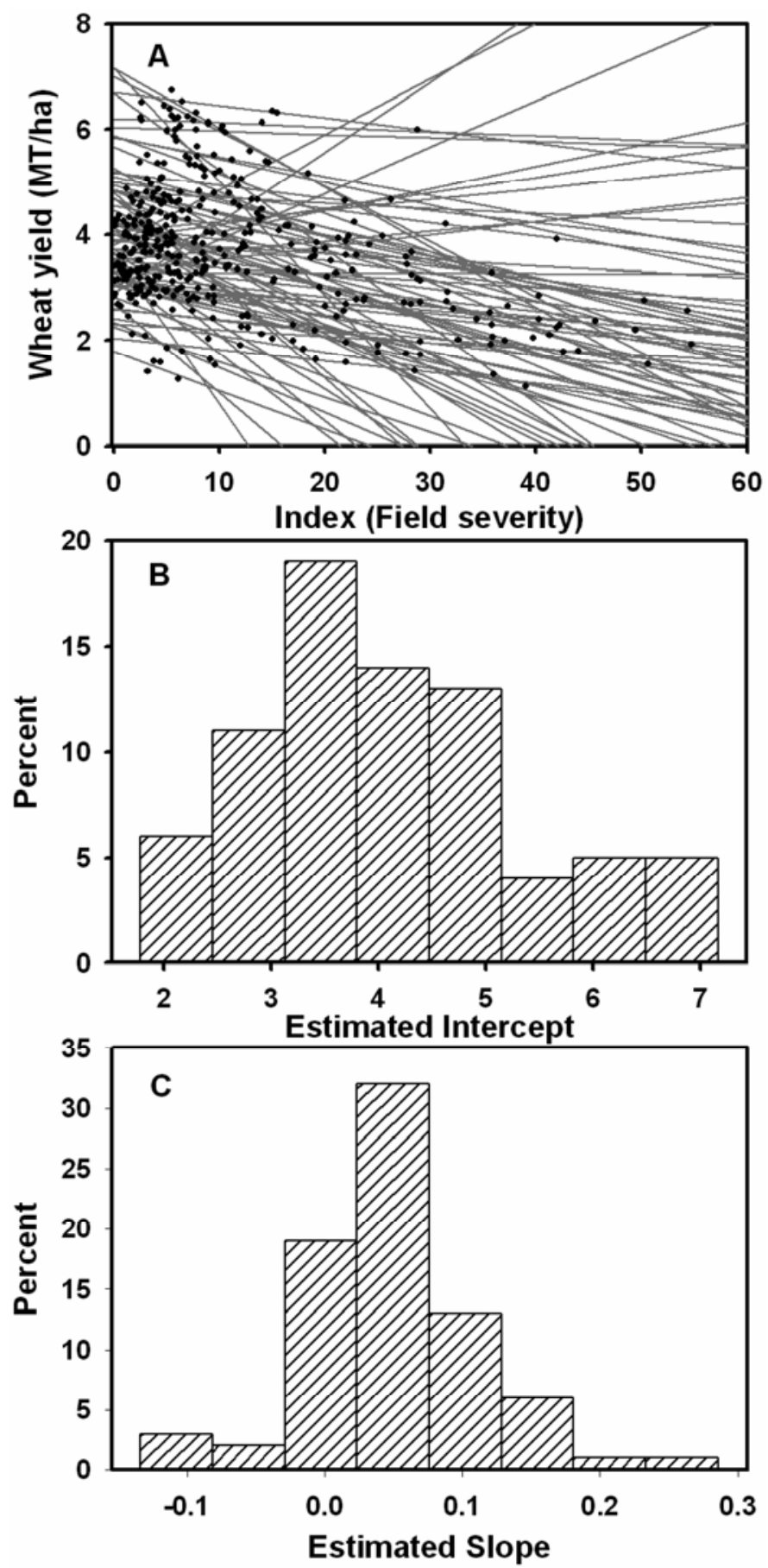

Fig. 2. Results for the fit of a simple linear model (equation 1b) to wheat yield $(W)$ in relation to Fusarium head blight index $(Y)$ for 77 studies. A, Observed $W$ values in relation to $Y$ (points), and predicted yields based on equation $1 \mathrm{c}$ (lines). Histogram of estimated $\mathbf{B}$, intercepts $\left(\hat{\beta}_{0 i}\right)$ and $\mathbf{C}$, slopes $\left(\hat{\beta}_{1 i}\right)$ of equation 1c. 
Random coefficient model. Equation 2 was successfully fitted to the data across 77 studies using maximum likelihood. Estimates of the population-average intercept and slope were $\hat{\beta}_{0}=$ $4.10 \mathrm{MT} / \mathrm{ha}(\mathrm{SE}=0.136)$ and $\hat{\beta}_{1}=0.038 \mathrm{MT} \mathrm{ha}^{-1} \%(\mathrm{SE}=$ $0.0030)$, respectively. Both estimated parameters were significantly different from $0(P<0.001)$. Estimates of the randomeffect variances and covariances, as well as the residual variance, were $\hat{\sigma}_{u_{0}}^{2}=1.35, \hat{\sigma}_{u_{1}}^{2}=8.5 \times 10^{-5}, \hat{\sigma}_{u_{0}, u_{1}}=-0.0042$, and $\hat{\sigma}_{e}^{2}=0.055$. Based on the between-study variance components, the amongstudy correlation of the effects of study on the intercept and slope was -0.40 .

The predicted study-specific intercept $\left(\hat{b}_{0 i}=\hat{\beta}_{0}+\hat{u}_{0 i}\right)$ and slope $\left(\hat{b}_{1 i}=\hat{\beta}_{1}+\hat{u}_{1 i}\right)$ were determined from the estimated BLUPs of the study-specific random effects together with the population-average parameters (equations $4,6 \mathrm{~b}$, and $6 \mathrm{c}$ ). The $\hat{b}_{0 i}$ values ranged from 1.63 to $6.90 \mathrm{MT} / \mathrm{ha}$ (Fig. 3C), and the ID was $3.09 \mathrm{MT} / \mathrm{ha}$. The range and spread among studies were similar to that found for the estimated intercepts when the simple linear equation $1 \mathrm{~b}$ was fitted to the data. Moreover, the estimated population-average intercept $\left(\hat{\beta}_{0}\right)$ was very similar to the mean of the estimated intercepts obtained when equation $1 \mathrm{~b}$ was used. Note that the mean of the study-specific $\hat{b}_{0 i}$ values is not a relevant term to calculate because the population-average $\hat{\beta}_{0}$ is the key central measure of the intercepts.

The $\hat{b}_{1 i}$ slope values ranged from 0.028 to $0.047 \mathrm{MT} \mathrm{ha}^{-1} \%^{-1}$ (Fig. 3D), with ID equal to 0.011 . This range and spread were considerably smaller than the values obtained for the estimated slopes when simple equation $1 \mathrm{~b}$ was fitted to the same data (Fig. 2C). There also were no predicted slopes with negative sign.
Thus, accounting for the random effects of studies resulted in 'shrinkage' of the study-specific slopes towards the estimated population-average slope (0.038). Such shrinkage in one or more of the parameters is typical with random coefficient models $(2,4,10)$.

Figure 3A shows the observations, the population-average predictions of yield (equation 6a), and the study-specific predictions of yield (equation $6 \mathrm{c}$ ). There are 77 study-specific prediction lines corresponding to the 77 studies. Each of these lines can be considered generally as a random sample from the population of lines depicting the relationship between wheat yield and disease index. The same population-average and study-specific lines are shown in Figure 3B, together with the $95 \%$ confidence interval for expected population-average yield; the individual data points were omitted to avoid clutter. A comparison of Figures $3 \mathrm{~B}$ and $2 \mathrm{~A}$ demonstrates the shrinkage of calculated slopes for the former.

Plots of the unconditional and conditional studentized residuals versus predicted yields (10) showed no evidence of patterns or outliers (L. V. Madden and P. A. Paul, unpublished data). This indicated that equation 2 was a reasonable description for the disease-yield relationship and that there was no graphical evidence against a linear relationship between $W$ and $Y$. Furthermore, there was a straight-line relationship between the studentized residuals and the normal scores in a normal-probability plot, indicating that the assumption of normality was reasonable. The graphical results for residuals were confirmed by fitting the more general equation 8 to the data. An $F$ test revealed that the disease factor $\Delta$ was not significant $(P>0.20)$, indicating that simpler linear equation 2 can be used instead.
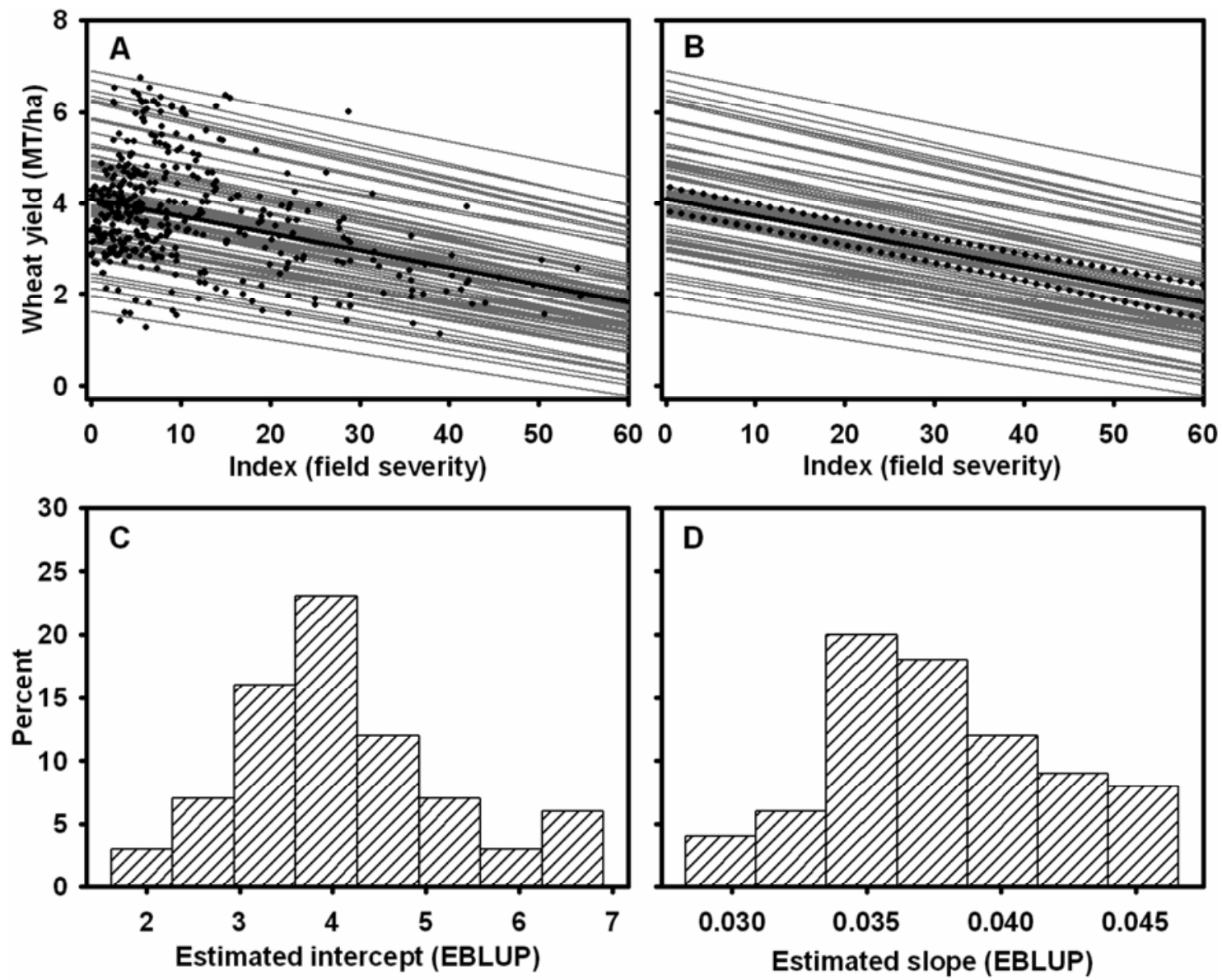

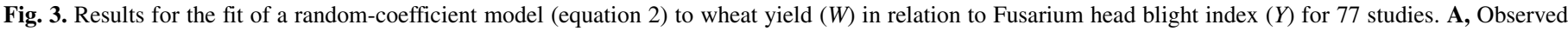

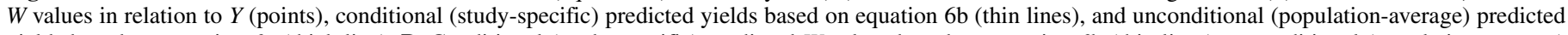

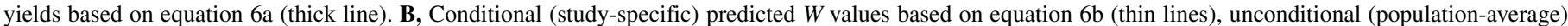

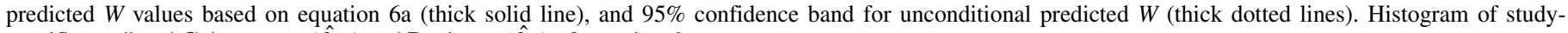
specific predicted $\mathbf{C}$, intercepts $\left(\hat{b}_{0 i}\right)$ and $\mathbf{D}$, slopes $\left(\hat{b}_{1 i}\right)$ of equation $6 \mathrm{c}$. 
The $R_{F}^{2}$ statistic (a unitless measure of the closeness of the population-average predictions to the yield data) was 0.136 . This low value is consistent with Figure $3 \mathrm{~A}$, where the large scatter of points around thick solid line ( $\hat{W}_{i j}$; equation $6 \mathrm{a}$ ) can be seen. The $R_{T}^{2}$ statistic (a unitless measure of the closeness of the studyspecific predictions to the yield data) was 0.968 . Furthermore, the concordance correlation coefficient was 0.983 . These large statistic values show that the individual study-specific lines came very close to the observed yields across all the individual studies.

Model expansion. The effect of wheat class on the diseaseyield relationship was assessed by fitting equation 7 to the data. Based on a Student's $t$ test, $\varsigma$ (effect of wheat type on the slope) was not significantly different from $0(P>0.30)$; this indicates that the slope was not affected by spring versus soft-red winter wheat. However, a $t$ test showed that $\xi$ (effect of wheat type on the intercept) was significantly different from $0(P<0.01)$; this indicates that the intercept depended on whether wheat was of the spring or soft-red winter type. Estimated parameters were: $\hat{\beta}_{0}=$ $4.57 \mathrm{MT} / \mathrm{ha}(\mathrm{SE}=0.188), \hat{\beta}_{1}=0.037 \mathrm{MT} \mathrm{ha}^{-1} \%^{-1}(\mathrm{SE}=0.0029)$, and $\hat{\xi}_{\text {spring }}=-0.85 \mathrm{MT} / \mathrm{ha}$ (i.e., $k=$ 'Spring') (SE =0.239). By definition, using the model syntax of the MIXED procedure of SAS, the estimate of the 'last' level of $\xi$ is 0 (i.e., $\hat{\xi}_{\text {Winter }} \equiv 0$, with $k=$ 'Winter'). Because of the negative value for $\hat{\xi}_{\text {Spring }}$, spring wheat cultivars had, on average, a $0.85 \mathrm{MT} / \mathrm{ha}$ lower yield than did soft red winter wheat cultivars.

As a generalization/expansion of equation $6 \mathrm{a}$, the populationaverage predictions of yield can be thus written as

$$
\hat{W}_{i j k}=\hat{\beta}_{0}+\hat{\xi}_{k}-\hat{\beta}_{1} Y_{i j k}
$$

which gives the following for spring and winter wheat:

$$
\begin{aligned}
\hat{W}_{i j \text { Spring }} & =4.57-0.85-0.037 Y_{i j \text { Spring }} \\
& =3.72-0.037 Y_{i j \text { Spring }} \\
\hat{W}_{i j \text { Winter }} & =4.57+0-0.037 Y_{i j \text { Winter }} \\
& =4.57-0.037 Y_{i j \text { Winter }}
\end{aligned}
$$

The study-specific predictions are obtained simply by adding $\hat{u}_{0 i}$ and $\hat{u}_{1 i}$ to the right side of equations $10 \mathrm{a}$ and $10 \mathrm{~b}$ (equation $6 \mathrm{~b}$ provides a simpler example):

$$
\begin{gathered}
\hat{W}_{i j \text { Spring }} \mid u_{0 i}, u_{1 i}=\left(3.72+\hat{u}_{0 i}\right)-\left(0.037+\hat{u}_{1 i}\right) Y_{i j \text { Spring }} \\
\hat{W}_{i j \text { Winter }} \mid u_{0 i}, u_{1 i}=\left(4.57+\hat{u}_{0 i}\right)-\left(0.037+\hat{u}_{1 i}\right) Y_{i j \text { Winter }}
\end{gathered}
$$

The study-specific (equations 11a and 11b) and populationaverage (equations 10a and 10b) predictions are shown in Figure $4 \mathrm{~A}$, together with the $95 \%$ confidence intervals for expected yields. The predicted study-specific intercepts $\left(\hat{b}_{0 i}\right)$ ranged from 1.59 to 7.41 MT/ha, with ID = $3.09 \mathrm{MT} /$ ha (Fig. 4B). The predicted study-specific slopes $\left(\hat{b}_{1 i}\right)$ ranged 0.021 to $0.053 \mathrm{MT} \mathrm{ha}^{-1} \%^{-1}$, with ID $=0.008$ (Fig. 4C). These summary statistics were very similar to those found when wheat type was not considered (see above). However, the consideration of wheat type did reduce the ID for the study-specific slope by $27 \%$ (from 0.011 down to 0.008).

Estimates of the random-effect variances and covariances, as well as the residual variance, were: $\hat{\sigma}_{u_{0}}^{2}=1.27, \hat{\sigma}_{u 1}^{2}=1.0 \times 10^{-4}$, $\hat{\sigma}_{u_{0}, u_{1}}=-0.0074$, and $\hat{\sigma}_{e}^{2}=0.054$. The among-study correlation was estimated as -0.65 . As judged by the magnitude of the AIC statistic, equations $11 \mathrm{a}$ and $11 \mathrm{~b}$ (with the estimated variance and covariance terms given here) fitted the data better (AIC $=348.3$ ) than models with: (i) a 0 for the among-study covariance of slope and intercept (AIC = 349.9); (ii) a 0 for the among-study covariance and a 0 for the among-study variance of the slopes $(\mathrm{AIC}=349.0)$; or (iii) a 0 for the among-study covariance and a 0 for the among-study variance of the intercepts (AIC $=844.2$ ). Note that situation ii is for a model with identical slopes for all studies (but varying intercepts), and situation iii is for a model with identical intercepts for all studies (but varying slopes). Comparisons of AIC values were not done with simpler randomcoefficient equation 2 without the wheat-class term, because relevant fixed effects (such as the effect of disease index and wheat class on yield) should be included in the model before simplifications to the random-effect structure are considered (10).
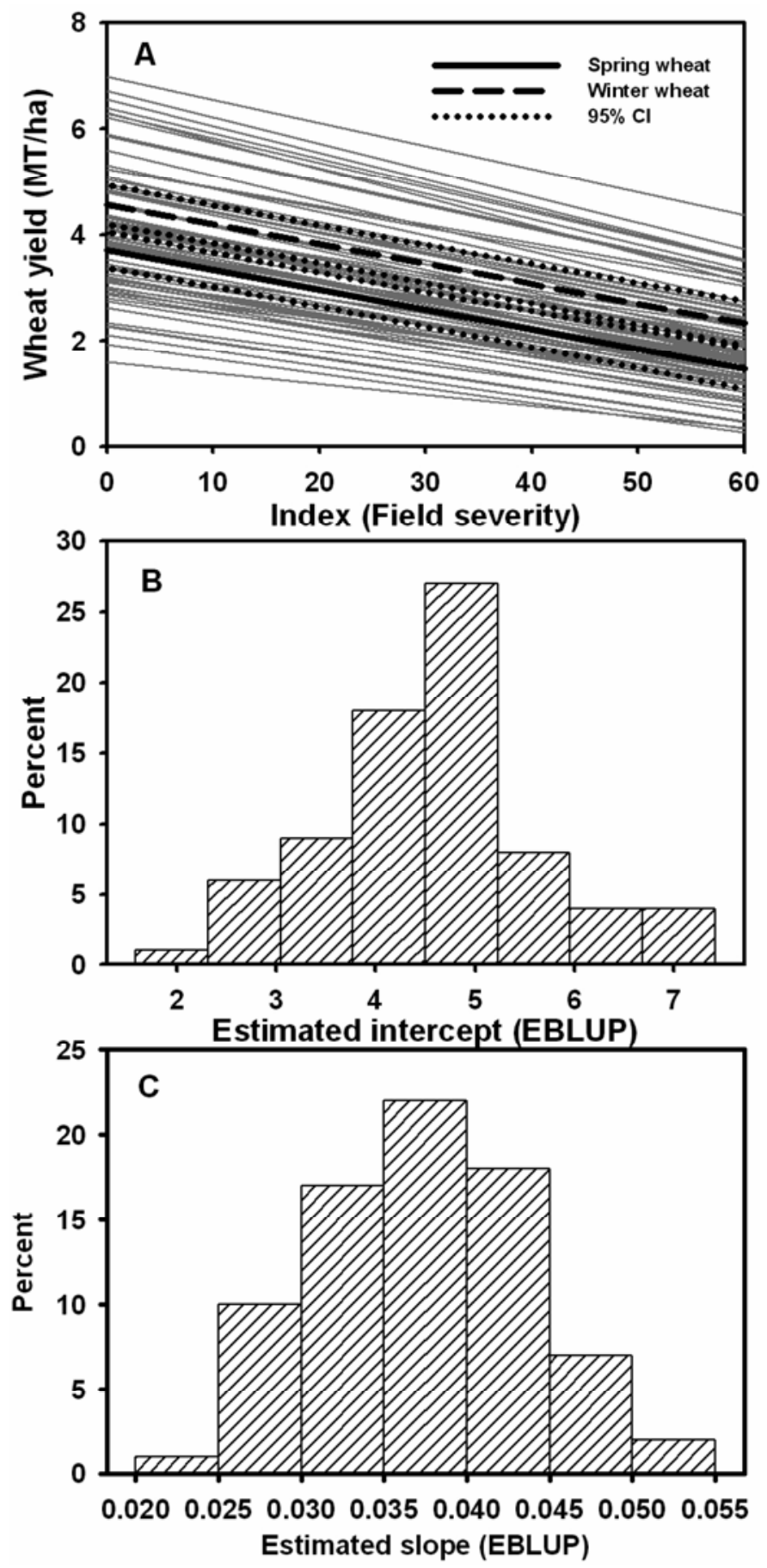

Fig. 4. Results for the fit of a random-coefficient model (equation 7, without the $\varsigma$ term) to wheat yield $(W)$ in relation to Fusarium head blight index $(Y)$ and wheat class for 77 studies. A, Conditional (study-specific) predicted $W$ values based on equations 11a and 11b (thin lines), unconditional (populationaverage) predicted $W$ values for spring and soft-red winter wheat based on equations 10a and 10b (thick lines), and 95\% confidence bands for unconditional predicted $W$ (thick dotted lines). Histogram of study-specific predicted $\mathbf{B}$, intercepts $\left(\hat{b}_{0 i}\right)$ and $\mathbf{C}$, slopes $\left(\hat{b}_{1 i}\right)$ of equations $11 \mathrm{a}$ and $11 \mathrm{~b}$. 
Plots of the conditional studentized residuals versus predicted yields showed a random pattern (Fig. 5A), and there was no evidence of obvious outliers. The normal-probability plot of the conditional studentized residuals indicated a straight line (Fig. $5 \mathrm{~B})$. These are graphic indications that the linear mixed model with assumed normally-distributed random-effect terms provided a reasonable representation of the data. The $R_{F}^{2}$ statistic (for population-average predictions) was 0.195 . This is slightly higher than the statistic when wheat class was not considered $(0.136)$.
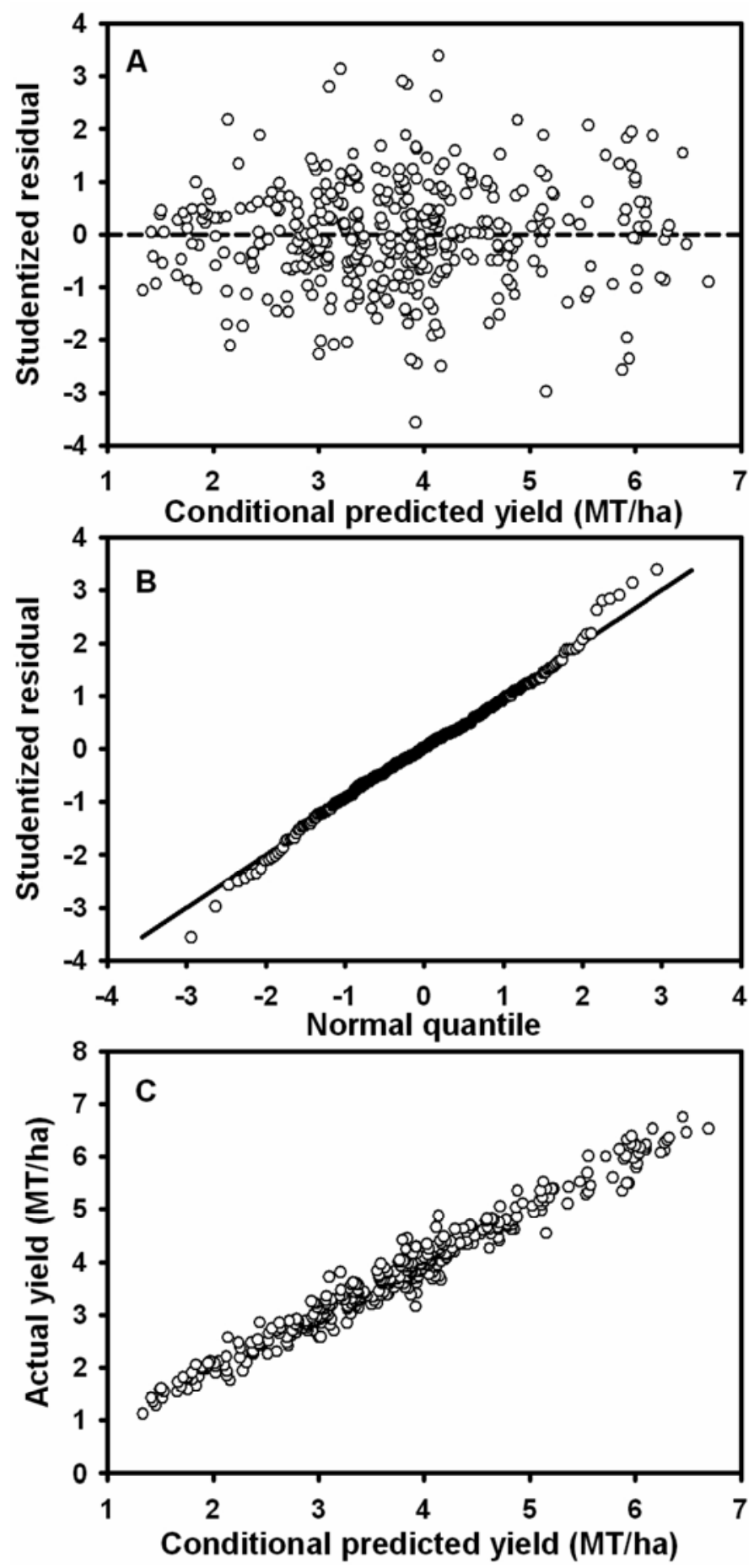

Fig. 5. A, Conditional studentized residuals (10) versus predicted yields for the random-coefficient model with disease index and wheat class as fixed effects (equations 11a and 11b). B, Normal-probability (quantile) plot (conditional studentized residuals versus normal scores) for the fit of equations 11a and 11b. C, Plot of observed yields versus conditional (study-specific) predicted yields for equations 11a and 11b. Studentized residuals are calculated by dividing the residuals by their estimated standard deviations (33).
The $R_{T}^{2}$ statistic (for study-specific predictions) was 0.968 , and the concordance correlation for the study-specific predictions coefficient was 0.984 . The good fit of the data for individual studies can be further seen by the plot of observed and studyspecific predictions of yield across all studies (Fig. 5C). The plot of the unconditional studentized residuals versus the predicted yields also had a random pattern, although there was lower variability at low values of predicted yield compared with the variation at high values of predicted yield (L. V. Madden and P. A. Paul, unpublished data).

Prediction, inverse prediction, and relative yield. One can use the random-coefficient results of equations 10 or 11 to predict the yield (or yield loss) for any particular study (equations 11a and $11 \mathrm{~b}$ ) or across all studies (equations 10a and 10b). One can also rearrange the equations to predict the value of disease index where a certain yield (or yield loss) is expected to occur. The latter is known sometimes as inverse prediction. The value of $Y$ at which $W$ is reduced by a fixed amount is determined by rearranging the prediction equation to solve for $Y$. Working generically with population-average prediction equation 9 , and suppressing the study $(i)$ and observation $(j)$ subscripts on $Y$ and $W$ for convenience, one can write

$$
\hat{Y}^{*}=\frac{\hat{W}^{*}-\hat{\beta}_{0}-\hat{\xi}_{k}}{-\hat{\beta}_{1}}
$$

for the predicted disease index at any chosen predicted yield (signified with the asterisk).

Figure 6A shows the predicted yields based on equations 10a and $10 \mathrm{~b}$ for spring and soft-red winter wheat. We calculate here the predicted disease at which yield is reduced by $1 \mathrm{MT} / \mathrm{ha}$; that is, the disease index where yield is reduced from 4.57 to 3.57 $\mathrm{MT} / \mathrm{ha}$ for winter wheat, and from 3.72 to $2.72 \mathrm{MT} / \mathrm{ha}$ for spring wheat. Based on equation 10b, and substitution of 3.57 for $\hat{W}^{*}$ in equation 12 , one obtains $\hat{Y}^{*}=(3.57-4.57-0) /(-0.037)=27 \%$ for winter wheat. Substitution of 2.72 for $\hat{W}^{*}$ in equation 12 , one obtains $\hat{Y}^{*}=(2.72-4.57-(-0.85)) /(-0.037)=27 \%$ for spring wheat. Because the slopes are the same for the two wheat classes, the predicted disease index is the same for a given absolute reduction in yield. The estimated SE for this estimated disease value was 2.20, which was determined based on delta method (3), which utilizes the estimated variances and the covariance. These calculations can also be performed with the study-specific intercepts and slopes.

In order to consider yield (or yield loss) on a relative scale, one divides both sides of equation 9 by $\hat{\beta}_{0}+\hat{\xi}_{k}$, which is the predicted yield when $Y=0$ (Chapter 12 in citation 16). The relative yield is labeled as lowercase $w\left(\hat{w}=\hat{W} /\left(\hat{\beta}_{0}+\hat{\xi}_{k}\right)\right)$. Multiplying by 100 gives the percent yield as a function of disease index:

$$
100 \hat{w}_{i j k}=100-\left(\frac{100 \hat{\beta}_{1}}{\hat{\beta}_{0}+\hat{\xi}_{k}}\right) Y_{i j k}
$$

Specific population-average results for spring and soft-red winter wheat, respectively, are

$$
\begin{aligned}
& 100 \hat{w}_{i j \text { Spring }}=100-0.99 Y_{i j \text { Spring }} \\
& 100 \hat{w}_{i j \text { Winter }}=100-0.81 Y_{i j \text { Winter }}
\end{aligned}
$$

Because the height of the yield line (in absolute units) depends on wheat class $\left(\beta_{0}+\xi_{k}\right)$, the slope of the relative-yield line depends on wheat class, even though the slope for absolute yield does not. In particular, the rate of decline in relative yield with increasing disease index is greater for spring wheat $\left(0.99 \%{ }^{-1}\right)$ than for soft red winter wheat $\left(0.81 \%^{-1}\right)$. This is because, for instance, $1 \mathrm{MT} / \mathrm{ha}$ is a larger percentage of 3.72 than of 4.57 . The relationship is demonstrated graphically in Figure 6B. At $Y=60 \%$ (about the 
highest disease index found), relative yield is predicted to be $40.6 \%$ for spring wheat and $51.4 \%$ for winter wheat.

Using equations $14 \mathrm{a}$ and $14 \mathrm{~b}$ (or equation 13), one can use inverse-prediction techniques to predict the level of disease index where a certain percentage loss will occur. Based on the population-average results, one obtains (suppressing subscripts again)

$$
\hat{Y}^{*}=\frac{100 \hat{w}^{*}-100}{-\left(100 \hat{\beta}_{1} /\left(\hat{\beta}_{0}+\hat{\xi}_{k}\right)\right)}
$$

The denominator of equation 15 is simply the slope of equation 13. The use of equation 15 is demonstrated for a yield reduction of $20 \%$; that is, we predicted the level of disease index at which relative yield is reduced from 100 to $80 \%$ (so that $\hat{w}^{*}=80 \%$ ). One obtains predicted disease values of $24.7 \%(\mathrm{SE}=1.96)$ for winter wheat and $20.0 \%(\mathrm{SE}=1.57)$ for spring wheat. The calculations are shown graphically with the thin broken vertical and horizontal lines in Figure 6B. With the estimated parameters found here, a given percentage loss of yield is predicted to occur at a lower disease index (on average) for spring wheat than for soft-red winter wheat, even though a given absolute loss of yield is predicted to occur at the same disease index (on average) for both wheat classes. These calculations can also be done with studyspecific intercepts and slopes.

\section{DISCUSSION}

James (6) made a strong argument 35 years ago that crop loss studies should be done, when possible, at multiple locations or in multiple years, in order to determine the range of possible relationships between yield (or yield loss) and disease intensity (such as disease incidence or severity [index]). However, due to the costs of conducting experiments and short time limits on funding, most published crop loss studies are based on results from a small number of locations and/or years $(16,32,36)$. Because of the high economic importance of FHB of wheat $(17,23,34,40)$, there have been considerable resources committed over more than a decade by the USWBSI to study the effects of fungicides on disease, DON, and to a lesser extent, wheat yield $(26,27,28)$. The studies involving yield provide a valuable data base to characterize the overall relationship between wheat yield and symptoms of FHB on wheat spikes (through the population-average model results), and also characterize the heterogeneity of the relationship (through the study-specific model results) for generally susceptible cultivars grown using standard agronomic practices for each location.

Surprisingly, there have been few studies that have explicitly characterized in a quantitative manner the functional relationship between symptoms of FHB and crop yield $(23,31,39)$ for wheat or other susceptible grain crops. We have found here that there was strong evidence for a straight-line linear relationship between yield $(W)$ and disease index $(Y)$, but that there was also significant variation in aspects of the relationship among studies, especially in terms of the height of the regression lines. Other studies have found either a straight-line or a curvilinear relationship for FHB and yield (23). Recently, Willocquet et al. (39) analyzed the relative-yield-loss data for a range of treatments reported in Mesterházy et al. $(18,19)$, and found a straight-line relationship between percentage loss and FHB disease symptoms for winter wheat grown in Hungary. They found a slope of 1.1, similar to the estimated slopes of 1.0 and 0.8 in our population-average equations $14 \mathrm{~b}$ and $14 \mathrm{a}$ for relative yield. Our analysis, as well as the analysis in Willocquet et al. (39), was based on treatment means for $Y$ and $W$, and it is known that use of averages (either from samples within plots/fields, or across plots) can reduce underlying curvilinear or nonlinear functional relationships that occur at a different (lower) spatial scale, especially if the disease is highly clustered (16).
The major factor identified so far influencing the heterogeneity in the relationship was wheat class. In particular, the predicted level of $W$ when $Y$ was 0 , or equivalently, the height of the prediction line, was significantly higher (by $0.85 \mathrm{MT} / \mathrm{ha}$, on average) for soft-red winter than for spring wheat. This is not surprising, because soft-red winter wheat typically has higher yield than spring wheat. Wheat class did not influence the slope of the line (in terms of yield in absolute units), indicating that there is no evidence from this analysis that FHB differently affected the yield response to increasing levels of disease index.

A naïve analysis based on simple linear regression (equation 1b) - which does not account for the random effects of study on the relationship (through any of the of model parameters) - clearly showed substantial variability in slopes as well as intercepts, with both positive and negative signs for the slope estimates. For the estimated slopes, histograms (Fig. 2C) clearly showed central nonzero values, implying that there was an overall nonzero average rate of decline in $W$ with unit increase in $Y$. Although a metaanalysis (29) could have been undertaken at this stage (based on the parameter estimates in Figure 2B and C), random-coefficient mixed modeling was used in this investigation because it allowed us to focus on direct characterization of the relationship between yield and disease, both overall (i.e., the population-average results) and in individual studies (i.e., the study-specific results),
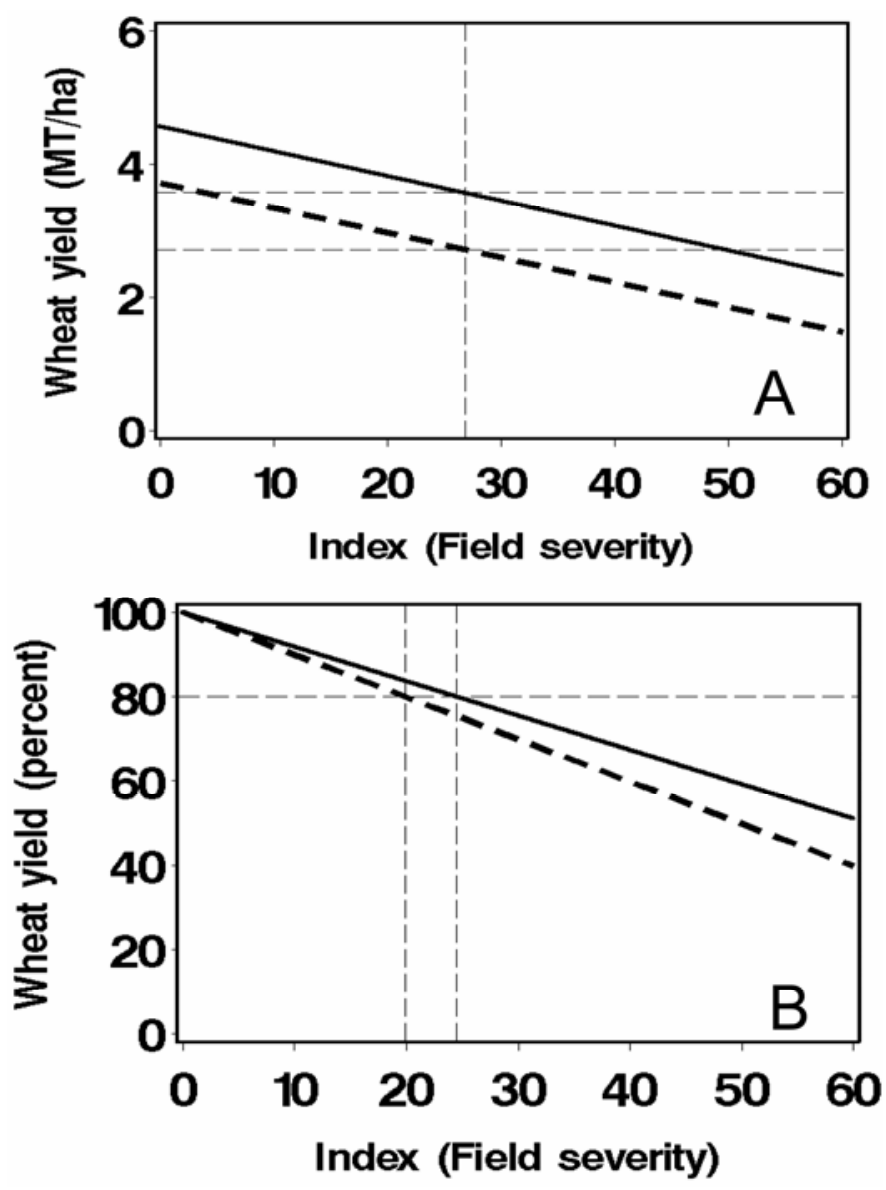

Fig. 6. A, Unconditional (population-average) predictions of wheat yield $(W)$ in relation to Fusarium head blight index $(Y)$ for spring (dashed line) and softred winter (solid line) wheat, based on equations 10a and 10b. Thin horizontal lines indicate the predicted yields corresponding to a $1 \mathrm{MT} / \mathrm{ha}$ reduction from the disease-free yield $\left(\hat{\beta}_{0}+\hat{\xi}_{k}\right.$ in equation 9$)$. Thin vertical line is the predicted disease index corresponding to the $1 \mathrm{MT} / \mathrm{ha}$ reduction in yield. $\mathbf{B}$,

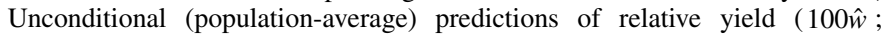
equations $14 \mathrm{a}$ and $14 \mathrm{~b}$ ) in relation to $Y$, for both spring (dashed line) and softred winter (solid line) wheat. Thin horizontal line indicates a $20 \%$ reduction in relative yield, and thin vertical lines indicate the predicted $Y$ where this reduction will occur for spring and winter wheat. 
and to explicitly account for the random effect of study on the relationship $(2,10)$. One consequence of random-coefficient modeling, compared to the naïve analysis (equation $1 \mathrm{~b}$ ), is the potential reduction ('shrinkage') in the values obtained for the studyspecific intercepts and slopes $(4,12)$. As stated by de Leeuw and Kreft (4), this shrinkage (which depends on the among-study variability of the data) is one of the primary advantages of random-coefficient models: study-specific predicted intercepts and slopes are more stable, and more plausible, than the separate (imprecise) estimates of the parameters obtained from simpler models such as equation $1 \mathrm{~b}$. The stability is because each studyspecific prediction is 'informed' by the results in all the other studies through the variability among studies.

Results from the fitting of a random-coefficient model to the data indicated that there was a very strong linear relationship between yield and disease index for the individual studies, as demonstrated by the high $R_{T}^{2}$ statistic of Liu et al. (11) for the study-specific predictions of yield, but that there was high variation in the relationship among studies. The latter is demonstrated by the small $R_{F}{ }^{2}$ statistic of Liu et al. (11) and the wide scatter of observations and study-specific predictions of yield around the population-average predictions of yield. Results clearly showed that the major effect of study was on the intercept of the diseaseyield model, although both intercept and slope varied with study. This can be seen in an ad hoc manner by looking at the ratio of the ID of the study-specific predicted slopes and intercepts $\left(\hat{b}_{0 i}, \hat{b}_{1 i}\right)$ to the population-average parameter estimates $\left(\hat{\beta}_{0}, \hat{\beta}_{1}\right)$, and multiplying by 100 . Based just on the model results without the wheat-class term, the relative variation of the slope was 29\% (100 $\times 0.011 / 0.038)$, whereas the relative variation of the intercept was $75 \%(100 \times 3.09 / 4.1)$. The magnitude of the among-study estimated variances $\left(\hat{\sigma}_{u_{0}}^{2}, \hat{\sigma}_{u_{1}}^{2}\right)$ relative to the population-average parameter estimates further demonstrates the high variation in the intercepts. Similar statistics can be obtained by using the results from the model with the wheat class term (equations 9 to 11a and 11b). Moreover, in addition to the differences in relative variation, the very large variation in the estimated slopes from the naïve simple regression analysis (equation 1 b) was reduced ('shrunken') greatly by accounting for random variation through the randomcoefficient-based analysis, with a reduction in inderdecile range from 0.17 down to 0.011. In fact, based on the AIC statistics, the random-coefficient model with separate (but stable) study-specific slopes (i.e., $\hat{\sigma}_{u_{1}}^{2}>0$ ) provided only a slightly better fit than did a random-coefficient model with equal study-specific slopes (i.e., all $u_{1 i}=0$, or equivalently, $\hat{\sigma}_{u_{1}}^{2}=0$ ). Given that the difference in AIC between this latter (equal-slope) model and the best fitting one was less then 1.0, it would be a reasonable approximation (with only a small loss in accuracy) to use a model with a single slope across all studies. However, the general random-coefficient model fitted the data much better than a model with equal studyspecific intercepts (i.e., $\hat{\sigma}_{u_{0}}^{2}=0$ ), based on the AIC statistics. Plus, the variation in the intercepts from the naïve analysis was only slightly reduced by the use of the random-coefficient approach, with a reduction in ID from 3.30 down to $3.09 \mathrm{MT} / \mathrm{ha}$. A comparison of Figures 2 and 3 (or Fig. 4) can be used to see the same result graphically. These results suggest strongly that the heterogeneity in the relationship was mostly in terms of the overall study yield, a reflection of wheat class, and unrecorded differences in growing conditions, environment, study-specific crop production and harvesting practices, and so on.

The fairly consistent and robust relationship between visible disease symptoms and wheat yield can be seen by considering the study-specific prediction of yield at, for example, $20 \%$ disease index. Based on equation $6 \mathrm{c}$, these predictions are given by $\hat{b}_{0 i}-\left(\hat{b}_{1 i} \cdot 20\right)$, with a different slope and intercept for each study, and with high variation in the $\hat{b}_{0 i}$ values. However, the studyspecific prediction of the actual decline in yield from the nodisease case is given by $\hat{b}_{1 i} \cdot 20$. In other words, the highly vari- able study-specific intercepts do not affect the prediction of the change in yield that occurs when symptoms of the disease are visible. Therefore, unsurprisingly, one would not be able to reliably predict actual yield for a given study based just on disease index, but one could reliably predict how much yield is reduced (MT/ha) based on disease index symptoms. This does not translate into a reliable prediction of the percent reduction in yield, however, because the percent decline depends on yield when disease is not visible (the intercept; see equation 13), which is the highly variable portion of the random-coefficient model.

Using univariate and multivariate meta-analyses, Paul et al. $(26,27)$ previously showed the percent reduction in head blight disease index that can be expected when wheat is treated with various triazole fungicides. Using $Y^{(C)}$ to represent the mean disease index in the control and $Y^{(T)}$ to represent the mean index for a particular treatment, and suppressing subscripts for convenience, the mean percent control (relative reduction in disease compared with the control) is given by

$$
C=100 \cdot\left[Y^{(C)}-Y^{(T)}\right] / Y^{(C)}
$$

When wheat was treated with a single application of prothioconazole, mean $C$ was $48 \%$ (with a $95 \%$ confidence interval of 43 to $53 \%$ ). This can be translated into the percent increase in yield (I) by the use of a fungicide treatment, relative to not using a fungicide (the control), based on the population-average (equations $6 \mathrm{a}$ and $10 \mathrm{a}$ and $10 \mathrm{~b}$ ) or study-specific (equations $6 \mathrm{c}$ and $11 \mathrm{a}$ and $11 \mathrm{~b}$ ) model-fitting results. $I$ is written as

$$
I=100 \cdot\left[W^{(T)}-W^{(C)}\right] / W^{(C)}
$$

With the range of disease index values and the estimated parameters found in the current investigation, two conclusions can be made: (i) $I$ will be less than $C$; and (ii) $I$ will depend on $Y^{(C)}$ (i.e., a single value of $C$ does not translate to a single value of $I$ ). Consider the situation with $C=48 \%, Y^{(C)}=30 \%$, and thus $Y^{(T)}=$ $15.6 \%$. Using the model-fitting results without the wheat-class term, the population-average predicted yield is $W^{(C)}=4.1-$ $0.038 \cdot 30=2.96 \mathrm{MT} / \mathrm{ha}$ for the control and $W^{(T)}=4.1-0.038$. $15.6=3.507$ for the fungicide treatment (equation $6 \mathrm{a}$ ). This gives $I=100 \cdot(3.507-2.96) / 2.96=18.5 \%$. With the same value of $C$ $(48 \%)$, but with $Y^{(C)}=15 \%$, one obtains $I$ of only $7.8 \%$.

Based on the population-average parameter estimates, it can be shown after several algebraic steps (eliminating $Y^{(T)}$ by expressing this term as a function of $C$ and $Y^{(C)}$ ) that $I$ can be written directly as

$$
I=\frac{C Y^{(C)}}{\left(\hat{\beta}_{0} / \hat{\beta}_{1}\right)-Y^{(C)}}
$$

One can substitute $\hat{\beta}_{0}+\hat{\xi}_{k}$ for $\hat{\beta}_{0}$ when wheat class is considered, as in equations $10 \mathrm{a}$ and $10 \mathrm{~b}$; one can also substitute $\hat{b}_{0}$ for $\hat{\beta}_{0}$, and $\hat{b}_{1}$ for $\hat{\beta}_{1}$, if one is making study-specific predictions. With $C=48 \%$ and $Y^{(C)}=30 \%$, one directly obtains $I=18.5 \%$ from this equation for the population-average result, as found above by using the yield equation with two different values of disease. $I$ can only be greater than $C$ if $Y^{(C)}>\hat{\beta}_{0} /\left(2 \hat{\beta}_{1}\right)$. With the parameter estimates here, the right-hand side of this inequality gives the critical disease index of $54.0 \%$, which is about the largest index value found across all studies $(54.7 \%)$; thus, percentage yield increase was less than percentage reduction in disease index in general for this system. Incorporating wheat-class effects into the formula for $I$ gives critical disease index values (where $C>I$ ) of $61.8 \%$ for soft-red winter wheat and $50.3 \%$ for spring wheat, which are larger than, or about the same as, the largest observed index value. Moreover, the larger the disease index before treatment, the larger the percent increase in yield would be by using a fungicide (at a fixed $C$ and yield-model parameters). If the 
impact of disease was less (i.e., if $\beta_{1}$ was lower [closer to 0]), then $I$ would be smaller. Moreover, smaller values of disease-free yield $\left(\hat{\beta}_{0}\right.$ or $\hat{\beta}_{0}+\hat{\xi}_{k}$ ) result in larger values of $I$ (with other terms being fixed). Thus, treatment of spring wheat would produce, on average, a higher percentage increase in yield compared with winter wheat (for a given $C$ ).

It should be noted that the calculation of $I$ in equation $16-$ which involves the relative change in yield between any two disease index values (defined through $C$ ) - is more complicated than the calculation of relative yield in equations 13, 14a, and $14 \mathrm{~b}$. For the latter, the standardization is in terms of the diseasefree yield, and does not directly involve a yield difference. Both sets of calculations demonstrate, however, the sensitivity of the outcome to the disease-free yield (for a fixed value of the slope or other terms being considered [e.g., $C$ ]). For instance, the yield response on a relative scale (i.e., either the percentage decline in yield [equations 14a and 14b] with unit increase in disease index, or percentage increase in yield [equation 16] with a given percentage decline in disease), was higher for spring than for winter wheat, a direct consequence of the magnitude of $\hat{\beta}_{0}$ or $\hat{\beta}_{0}+\hat{\xi}_{k}$ when $\hat{\beta}_{1}$ does not vary with wheat class. It is possible, of course, that there are additional inherent differences between spring and winter wheat (other than overall magnitude of yield) that could be influencing the relative yield responses, but other hypotheses can not be tested with the available data.

In conclusion, through random-coefficient modeling, we found that there was a linear relationship between wheat yield and FHB disease index, and that there was high variability among studies in the height of the lines, and relatively low variability in the slope of the lines. Moreover, the linearity of the relationship and the stability of the slopes held across a wide range of disease-free yield predictions (from around 1.6 to $7 \mathrm{MT} / \mathrm{ha}$ ). Through the population-average estimates of intercept and slope in the fitted random-coefficient model, the significant 'global' relationship was characterized, demonstrating that expected yield was predicted to decline with increasing symptoms of the disease on spikes. Among other things, these results can be incorporated directly into the injury-profile models of Willocquet et al. (39) to assess strategically the overall impact of multiple pests and diseases in wheat production systems. In contrast, through the studyspecific predictions of intercept and slope in the fitted randomcoefficient model, the significant 'local' relationships (for different years and locations, and other study conditions) were characterized. The high variability found in study-specific intercepts precludes any reliable prediction of actual wheat yield based on the population-average results; however, because of the lower variability in the study-specific slopes, it would be more reasonable to predict the expected reduction in yield due to FHB, if a reliable estimate of disease index were available.

Several possible future investigations are suggested by the current results. First, the linearity of the disease-yield relationship can be confirmed (or refuted) if a fuller range of disease index values, and a larger total number of different index values, are obtained within individual studies. Second, it would be useful in future studies if some of the different levels of disease index (and resulting yield) are obtained without the use of fungicides, in order to determine if the fungicides themselves are affecting the realized disease-yield relationship (13). Third, because the data here were for susceptible cultivars primarily, it would be worthwhile to determine the extent to which cultivar resistance (or tolerance) to head blight affects the disease-yield relationship. Predictions of the relationship are clouded, however, by the complexity of resistance types known-or believed - to exist for this disease $(18,20)$, with a given cultivar possibly comprised of more than one form of resistance. We hypothesize that resistance to initial infection (Type I) or to fungal colonization of spikes, including the glumes (Type II), will affect yield primarily through a change in observed disease index (alteration of the predictor variable), and not by changes in the parameters of the model. Other forms of resistance, such as resistance to infection or colonization of the kernels (22), or tolerance (often classified as a form of "resistance" in the head blight literature), would not be manifested in changes in observed disease index. Rather, we hypothesize that these cultivar traits will affect the estimated slope of the relationship $(21,22)$. Since the ultimate goal of FHB management programs is to integrate (moderately) resistant cultivars and other controls (such as fungicide treatment, when needed [14]) in order to minimize yield and quality losses due to FHB, characterization of the disease-yield relationship for a range of cultivars with different putative types of resistance is of critical importance. For these future studies, either linear or nonlinear random-coefficient models will be of value in characterizing relationships when results are combined from multiple studies.

\section{ACKNOWLEDGMENTS}

We thank M. A. Draper, D. E. Hershman, P. E. Lipps, and M. P. McMullen for their previous collaboration on synthesis and analysis of data from the Uniform Fungicide Trials. We further thank all the researchers who conducted one or more of the fungicide trials. Salaries and research support were provided by state and federal funds to the Ohio Agricultural Research and Development Center (OARDC). This investigation is based upon work supported, in part, by the U.S. Department of Agriculture (Agreement No. 59-0790-4-112). This is a cooperative project with the U.S. Wheat \& Barley Scab Initiative (USWBSI). Any opinions, findings, conclusions, or recommendations expressed in this publication are those of the author(s) and do not necessarily reflect the view of the U.S. Department of Agriculture.

\section{LITERATURE CITED}

1. Bastiaans, L. 1991. Ratio between virtual and visual lesion size as a measure to describe reduction in leaf photosynthesis of rice due to leaf blast. Phytopathology 81:611-615.

2. Brown, H., and Prescott, R. 2006. Applied Mixed Models in Medicine. 2nd ed. John Wiley \& Sons, Chichester, UK.

3. Casella, G., and Berger, R. L. 2002. Statistical Inference. 2nd ed. Duxbury Press, NY.

4. de Leeuw, J., and Kreft, I. G. G. 1995. Questioning multilevel models. J. Educ. Behav. Stat. 20:171-189.

5. Garrett, K. A., Madden, L. V., Hughes, G., and Pfender, W. F. 2004. New applications of statistical tools in plant pathology. Phytopathology 94:999-1003.

6. James, W. C. 1974. Assessment of plant diseases and losses. Annu. Rev. Phytopathol. 12:27-48.

7. James, W. C., Teng, P. S., and Nutter, F. W., Jr. 1991. Estimated losses of crops from plant pathogens. Pages 15-51 in: CRC Handbook of Pest Management in Agriculture. D. Pimentel, ed. CRC Press, Boca Raton, FL.

8. Johnson, K. B. 1992. Evaluation of a mechanistic model that describes potato crop losses caused by multiple pests. Phytopathology 82:363-369.

9. Large, E. C. 1954. Growth stages in cereals. Plant Pathol. 3:128-129.

10. Littell, R. C., Milliken, G. A., Stroup, W. W., Wolfinger, R. D., and Schabenberger, O. 2006. SAS for Mixed Models. 2nd ed. SAS Institute, Cary, NC.

11. Liu, H., Zheng, Y., and Shen, J. 2008. Goodness-of-fit measures of $R^{2}$ for repeated measures mixed effect models. J. Appl. Stat. 35:1081-1092.

12. Longford, N. T. 1993. Random Coefficient Models. Oxford University Press, Oxford.

13. Madden, L. V. 1983. Measuring and modeling crop losses at the field level. Phytopathology 73:1591-1596.

14. Madden, L. V. 2006. Botanical epidemiology: Some key advances and its continuing role in disease management. Eur. J. Plant Pathol. 115:3-23.

15. Madden, L. V., Hughes, G., and Irwin, M. E. 2000. Coupling diseaseprogress-curve and time-of-infection functions for predicting yield loss of crops. Phytopathology 90:788-800.

16. Madden, L. V., Hughes, G., and van den Bosch, F. 2007. The Study of Plant Disease Epidemics. American Phytopathological Society, St. Paul, $\mathrm{MN}$.

17. McMullen, M., Jones, R., and Gallenburg, D. 1997. Scab of wheat and barley: A re-emerging disease of devastating impact. Plant Dis. 81:13401348.

18. Mesterházy, Á., Bartók, T., Kászonyi, G., Varga, M., Tóth, B., and Varga, J. 2005. Common resistance to different Fusarium spp. causing Fusarium head blight in wheat. Eur. J. Plant Pathol. 112:267-281. 
19. Mesterházy, Á., Bartók, T., and Lamper, C. 2003. Influence of wheat cultivar, species of Fusarium, and isolate aggressiveness on the efficacy of fungicides for control of Fusarium head blight. Plant Dis. 87:1107-1115.

20. Mesterházy, Á., Bartók, T., Mirocha, C. G., and Komoroczy, R. 1999. Nature of wheat resistance to Fusarium head blight and the role of deoxynivalenol for breeding. Plant Breeding 118:97-110.

21. Muthomi, J. W., Oerke, E.-C., Dehne, H.-W., and Mutitu, E. W. 2002. Susceptibility of Kenyan wheat varieties to head blight, fungal invasion and deoxynivalenol accumulation inoculated with Fusarium graminearum. J. Phytopathol. 150:30-36.

22. Nicholson, P., Gosman, N., Draeger, R., Thomsett, M., Chandler, E., and Steed, A. 2007. The Fusarium head blight pathosystem: Status and knowledge of its components. Pages 23-36 in: Wheat Production in Stressed Environments. H. T. Buck, J. E. Nisi, and N. Salomón, eds. Springer, Amsterdam, the Netherlands.

23. Parry, D. W., Jenkinson, P., and McLeod, L. 1995. Fusarium ear blight (scab) in small grain cereals-A review. Plant Pathol. 44:207-238.

24. Paul, P. A., El-Allaf, S. M., Lipps, P. E., and Madden, L. V. 2005. Relationships between incidence and severity of Fusarium head blight on winter wheat in Ohio. Phytopathology 95:1049-1060.

25. Paul, P. A., Lipps, P. E., De Wolf, E., Shaner, G., Buechley, G., Adhikari, T., Ali, S., Stein, J., Osborne, L., and Madden, L. V. 2007. A distributedlag analysis of the relationship between Gibberella zeae inoculum density on wheat spikes and weather variables. Phytopathology 97:1608-1624.

26. Paul, P. A., Lipps, P. E., Hershman, D. E., McMullen, M. P., Draper, M. A., and Madden, L. V. 2007. A quantitative review of tebuconazole effect on Fusarium head blight and deoxynivalenol content in wheat. Phytopathology 97:211-220.

27. Paul, P. A., Lipps, P. E., Hershman, D. E., McMullen, M. P., Draper, M. A., and Madden, L. V. 2008. Efficacy of triazole-based fungicides for Fusarium head blight and deoxynivalenol control in wheat: A multivariate meta-analysis. Phytopathology 98:999-1011.

28. Paul, P. A., Lipps, P. E., and Madden, L. V. 2005. Relationship between visual estimates of Fusarium head blight intensity and deoxynivalenol accumulation in harvested wheat grain: A meta-analysis. Phytopathology 95:1225-1236.

29. Paul, P. A., Lipps, P. E., and Madden, L. V. 2006. Meta-analysis of regression coefficients for the relationship between Fusarium head blight and deoxynivalenol content of wheat. Phytopathology 96:951-961.

30. Pimentel, D., Lach, L., Zuniga, R., and Morrison, D. 2000. Environmental and economic costs associated with non-indigenous species in the United States. BioScience 50:53-65.

31. Pirgozliev, S. R., Edwards, S. G., Hare, M. C., and Jenkinson, P. 2003 Strategies for the control of Fusarium head blight in cereals. Eur. J. Plant Pathol. 109:731-742.

32. Savary, S., Teng, P. S., Willocquet, L., and Nutter, F. W., Jr. 2006. Quantification and modeling of crop losses: A review of purposes. Annu. Rev. Phytopathol. 44:89-112.

33. Schabenberger, O., and Pierce, F. J. 2002. Contemporary Statistical Models for the Plant and Soil Sciences. CRC Press, New York.

34. Shaner, G. 2003. Epidemiology of Fusarium head blight of small grain cereals in North America. Pages 84-119 in: Fusarium Head Blight of Wheat and Barley. K. J. Leonard and W. R. Bushnell, eds. American Phytopathological Society, St. Paul, MN.

35. Stack, R. W. 2000. Return of an old problem: Fusarium head blight of small grains. Plant Health Progress. doi:10.1094/PHP-2000-0622-01-RV.

36. Teng, P. S. 1985. Construction of predictive models: II. Forecasting crop losses. Pages 179-206 in: Advances in Plant Pathology, Vol. 3; Mathematical Modelling of Crop Disease. C. A. Gilligan, ed. Academic Press, London.

37. Vonesh, E. F., Chinchilli, V. M., and Pu, K. 1996. Goodness-of-fit in generalized nonlinear mixed-effects models. Biometrics 52:572-587.

38. Waggoner, P. E., and Berger, R. D. 1987. Defoliation, disease, and growth. Phytopathology 77:393-398.

39. Willocquet, L., Aubertot, J. N., Lebard, S., Robert, C., Lannou, C., and Savary, S. 2008. Simulating multiple pest damage in varying winter wheat production situations. Field Crops Res. 107:12-28.

40. Windels, C. E. 2000. Economic and social impacts of Fusarium head blight: Changing farm and rural communities in the northern Great Plains. Phytopathology 90:17-21.

41. Wolfinger, R. D. 1996. Heterogeneous variance-covariance structures for repeated measures. J. Agric. Biol. Environ. Stat. 1:205-230.

42. Zadoks, J. C., Chang, T. T., and Konzak, C. F. 1974. A decimal code for the growth stages of cereals. Weed Res. 14:415-421.

43. Zadoks, J. C., and Schein, R. D. 1979. Epidemiology and Plant Disease Management. Oxford University Press, Oxford. 